\title{
SEAWAY AND OCTOPUS AS A STUDY TOOLS FOR SHIP MOTIONS
}

\author{
Manea Mihaela-Greti \\ Ovidius University of Constanta, \\ Faculty of Mechanical Enginering \\ Mamaia Ave., No. 124, 900527, Constanța, Romania \\ E-mail: mihaelagretimanea@gmail.com
}

\begin{abstract}
The engineers are interested in the theoretical and practical ways by which, in the activity of design, construction and operation of the ship, the complications generated by the sea waves can be overcome. The paper presents some examples regarding the possibilities of computer-assisted study of characteristics that define the behaviour of the ship under navigational conditions (linear and angular amplitudes of movements, energy spectre of response to different positions of the ship in relation to the incident waves). The study, carried out for a container vessel, took into account the different operating speeds of the ship and rudder angles. There were used features offered by two tools for modelling and simulation OCTOPUS and SEAWAY - software that allows the study of the computerized behaviour of the ship on the waves in the real navigational conditions. The program library was used for both the ship and navigation environment (modelled using Jonswap energy spectrum).
\end{abstract}

Keywords: ship motions, computer simulation, wave energy and RAO spectre, containership.

\section{INTRODUCTION}

The description of the interaction process between the body of the ship - a technical system of recognized complexity - and the real marine environment, respectively the behaviour that it generates, is one of the difficult problems of the ship's mechanics.

The various theoretical solutions, of the various researchers interested in this field, consist in the elaboration of mathematical models based on physics, which aims to describe as accurately as possible the problem addressed, responding to the practical needs of the shipbuilders and the navigating personnel.

A permanent desire is the validation of the theoretical models through practical tests in real sea (on prototypes), experiments in hydrodynamic laboratories (on models) and numerical simulations (computer assisted).
In this context, the present work aims to partly contribute to solving the problem related to the study of the behaviour of the ship under real navigation conditions, the related purpose being the highlighting of the numerical simulation performances with the help of OCTOPUS and SEAWAY software.

Representative bibliographic materials were consulted both nationally (Omnivore, L. [1]) and internationally. (Bhattacharyya, R., [2]; Edward, V., L., [3]; Fossen, T., [4]; Journey, J.M.J., [5]).

\section{SOME THEORETICAL ASPECTS}

The general equation of motion of the ship, in real sea, in vector version is [4]

$$
M_{\eta}(\eta) \ddot{\eta}+C_{\eta}(v, \eta) \dot{\eta}+D_{\eta}(v, \eta) \dot{\eta}+g_{\eta}(\eta)=\tau_{M \theta}+\tau_{\eta}
$$

When writing the equation, the following were taken into consideration: the analysis of the interdependent phenomena and the 
changes generated by external disturbing factors; using the principle of superposing effects in assessing the irregularity of the navigation environment; applying modern analytical mechanics to determining the matrices: the mass inertia of the ship, comple- mentary to the movement of the ship, the additional masses of water; adaptation and refinement of the strip theory for use in solving the problems raised by determining the hydrodynamic coefficients.

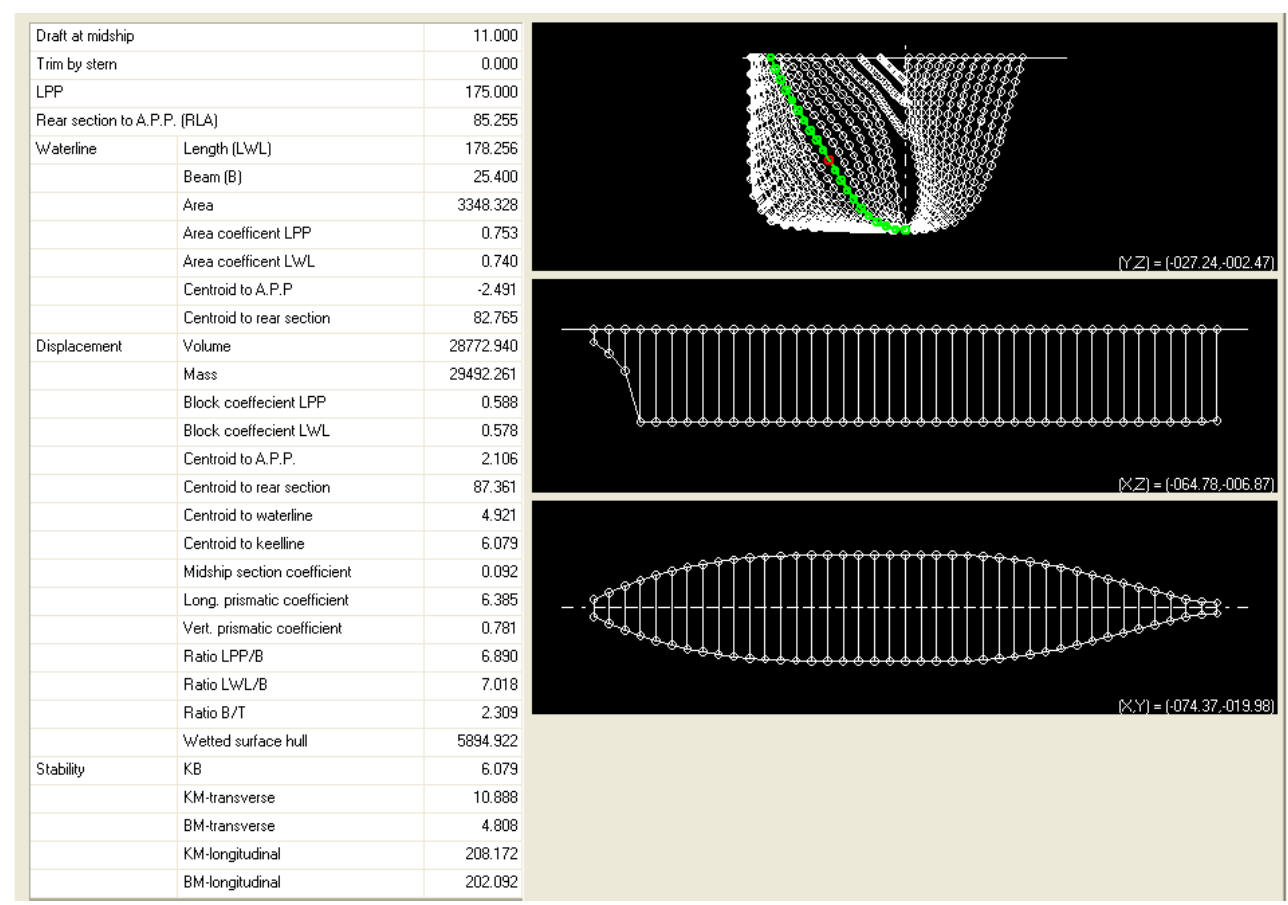

Fig. 1. The ship geometry

The study, carried out for the ship at full load (11 m draft), is conditioned by the possibility of loading, accurately, in the program library, the weights distribution diagram on board the ship.

It is also necessary to specify the navigation conditions, taking into account relevant elements:

- the navigation route (for the presented work, the route was chosen in the Mediterranean Sea;

- encountering angle (the angle below which the ship meets the wave front were chosen between $10^{\circ}$ and $35^{\circ}$ with a pitch of 5 $\left.5^{0}\right)$

- the diagram of the recorded waves (by specifying the significant wave height and the period of zero crossing).
It is necessary to observe that, in order to obtain an energy spectrum whose frequency of height variation complies with the theoretical law of Rayleigh distribution, OCTOPUS accepts that a recording of 100,000 waves is optimal.

The quality of the study is directly influenced by the existence of a wave spectrum specific to the navigation area, which must be uploaded by the user (the program does not contain such a library). Frequently is used theoretical wave energy spectra (the program has the Jonswap, Bretschneider and Pierson-Moscowitz models). For the study included in this paper, the Jonswap spectrum was used.

Fig. 2 shows the wave spectrum, as input size for the movement of the ship in a 
horizontal plane (yaw), corresponding to a

domain of $(0.2 \ldots 1.4) \mathrm{rad} / \mathrm{s}$ and for the speed variation of the encountering pulsation in the range considered $(12,5 ; 14,5 ; 16,5 \& 18,5) \mathrm{Knts}$.



Fig. 2. The Wave spectre for Yaw motion 
The ship is a system which, subject to some "input" actions, responds with other "output" actions, through a transfer function.
For the study of the movements that the ship executes on waves, this transfer function is called - Response Amplitude Operator - RAO.
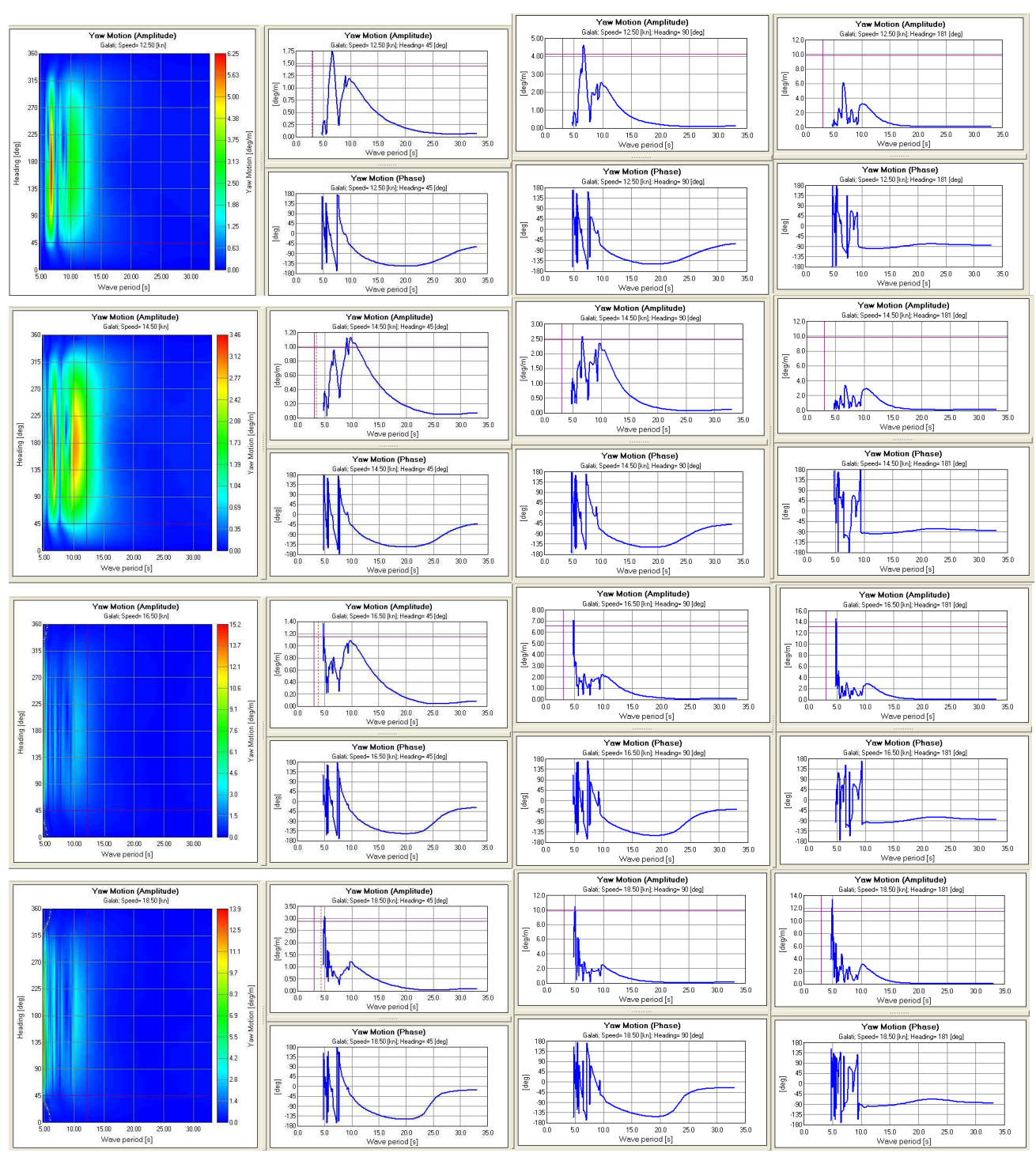

Fig. 3. The RAO for the yaw motion

The ship system, excited by waves, produces different responses for all the six degrees of freedom that it can have, as a free rigid body.
Fig. 3. illustrates the response spectrum for horizontal plane of the ship motion (yaw). 


\section{CONCLUSIONS}

On the problems with major interest for the marine engineers and the naval architects concerning the study of the general movements of the ship under real conditions of navigation, the ones regarding the worsening of the governance of the ship are noted, due to:

- the decrease in manoeuvrability, under the adverse hydrodynamic action of the waves on the rudder;

- the loss of route stability by the occurrence of the drift phenomenon caused by the unfavourable actions of the wind and the sea currents, when, for different reasons, the governing system can no longer function.

Another important problem concerns the diminution of the dynamic nautical qualities of march, due to:

- worsening the functioning of the propulsion system on the whole kinematic chain (the propellers come out of the water and rotate empty, at the re-entrance to the water being overloaded and generating shocks in the system);

- reducing the speed of the ship, as a result of the increase of advance ship resistance and malfunctions of the thrusters under turbulent water condition;

- reducing the autonomy of the ship by increasing energy consumption.

The verification of the ship's constructive and operating performance, as well as the validation of its nautical qualities by the method of the tests performed in real sea, on prototypes, implies extremely high costs and high construction times.

The study of the general movements of the ship under real navigation conditions is carried out, in a modern way, from the perspective of systems theory, taking into account the interdependence of the analyzed phenomena and the character of their modification under the action of disruptive factors.

Therefore, naval architects recommend the use of numerical methods of analysis and specialized software in anticipating the movements of the ship on the waves, follow- ing which the sea trials constitute a validation of these simulations.

The main purpose of the work was to highlight the performances that the OCTOPUS and SEAWAY software can offer in the design activity, even from the preliminary stages.

The exemplification was made for the movements of the ship on the horizontal plane and was completed by tracing the trajectories of the moving ship. (Fig. 4).

The vessel, chosen to exemplify the study, is a multifunctional container type, with a bulbous at the bow and the mirror aft .

The construction of the stern is suitable for equipping it with a semi-suspended, semicompensated rudder, with a hydrodynamic profile, posed in the centre plane of the ship. The ship is equipped with a propeller with 4 fixed blades, also arranged in the ship's diamteral plane. The speed of the ship was varied between 12.5 and 18.5 knots, the propeller shaft speed being between 80 and $150 \mathrm{rpm}$. The rudder was swinging toward the port side, from $10^{0}$ to $35^{\circ}$, with a step of $5^{0}$.

The total duration of the ship's gyration depends on the speed of the march and is approximately 3 to 5 minutes. The study here presented was performed for an analysis interval of 1000 seconds (considered propper in the analysis of the ship's behaviour).

The help of OCTOPUS and SEAWAY software was validated with data obtained after completing the sea trials.

After tying the rudder and keeping it at the desired angle, the centre of gravity of the ship, running at a certain speed, describes a curved trajectory.

The gyration diameter depends especially on the rudder angle, and it is even smaller as the rudder angle grows. For high speeds of the ship, the wide rudder angles should be avoided, since the transverse stability of the ship is affected, and, in extreme situations, the ship can be overturned.

For low speeds of the ship, steering the rudder at low angles (unless required by the operating situation of the ship) should be avoided, as the gyration diameter increases greatly. 

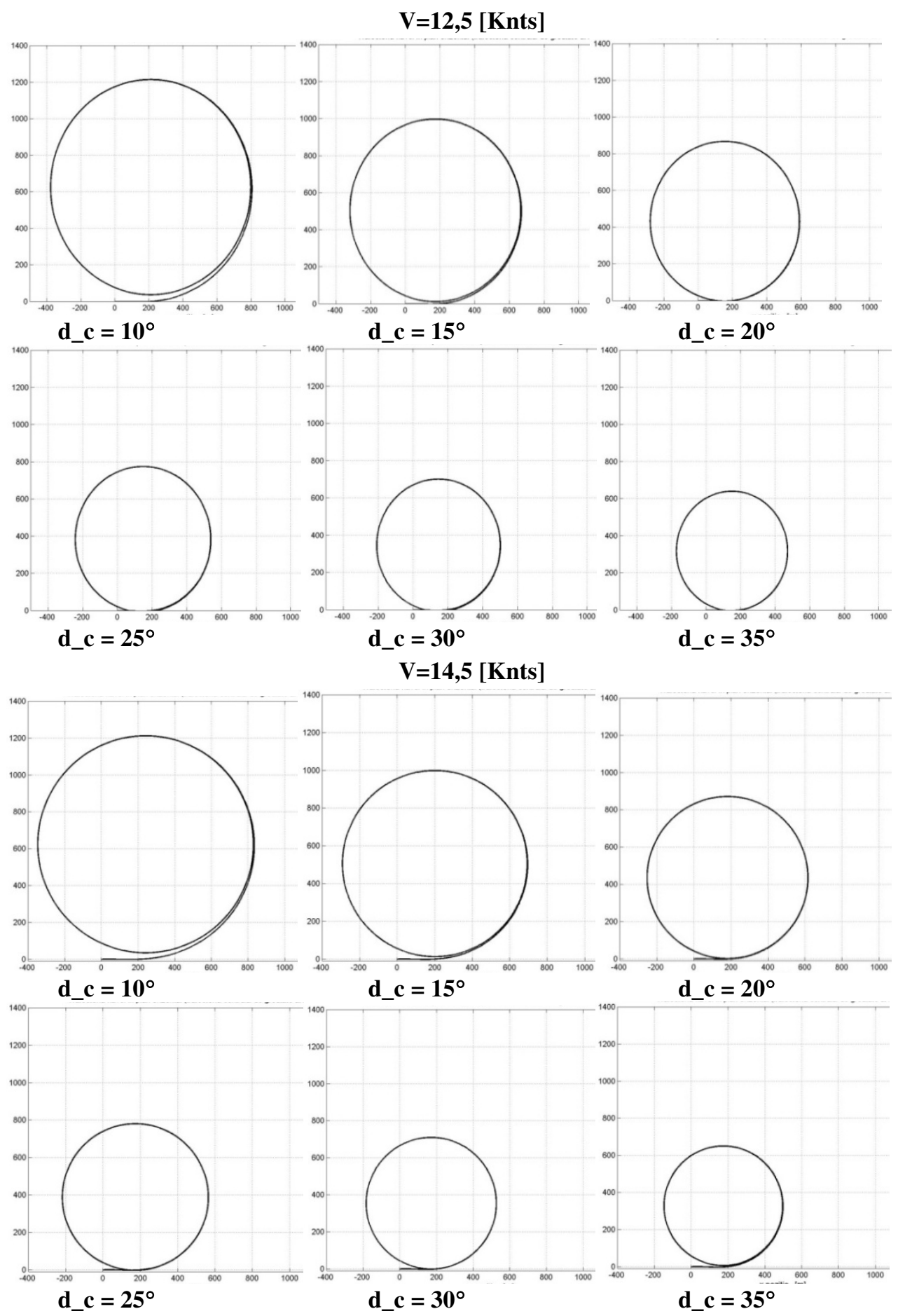

Fig. 4. The ship trajectory 



Fig. 4. The ship trajectory (continuation) 


\section{Acknowledgements}

Thanks to the University of Constanta for supporting the research carried out with the help of the OCTOPUS software.

\section{REFERENCES}

[1]. Domnisoru, L.,"Ship dynamics. Oscillations and vibrations", Technical Publishing House, Bucharest 2001.

[2]. Bhattacharyya, R., Dynamics of marine vehicles, New-York, John Wiley \& Sons, 1978.
[3]. Edward, V.L., Principles of naval architecture, Vol. I, II, III, New Jersey, The Society of Naval Architects and Marine Engineers, 1989.

[4]. Fossen, T., Guidance and control of ocean vehicles, University of Trondheim, Norway, John Wiley \& Sons, 1994.

[5]. Journee, J.M.J., Theoretical manual of SEAWAY, Delft University of Technology, Shiphydromechanics laboratory, Netherland, 2001.

Paper received on November $9^{\text {th }}, 2019$ 